\title{
Protein Kinase A Subunit $\alpha$ Catalytic and A Kinase Anchoring Protein 79 in Human Placental Mitochondria
}

\author{
Maggie Pui Chi $\mathrm{Ma}^{2}$ and Murray Thomson*,1 \\ ${ }^{1}$ School of Biological Sciences, The University of Sydney, NSW, 2006, Australia \\ ${ }^{2}$ Children’s Medical Research Institute, 214 Hawkesbury Rd, Westmead NSW 2145, Australia
}

\begin{abstract}
Components of protein phosphorylation signalling systems have been discovered in mitochondria and it has been proposed that these molecules modulate processes including oxidative phosphorylation, apoptosis and steroidogenesis.

We used electrophoresis and Western blots probed with specific antibodies to protein kinase A $\alpha$ catalytic subunit (PKA $\alpha$ Cat) and A kinase anchoring protein of approximately $79 \mathrm{kDa}$ molecular weight (AKAP79) to demonstrate the presence of these two proteins in human placental mitochondria. Heavy mitochondria characteristic of cytotrophoblast were separated from light mitochondria characteristic of syncytiotrophoblast by centrifugation. PKA $\alpha$ Cat and AKAP79 were present in both heavy and light mitochondria with no significant difference in concentration. Sucrose density gradient separation of submitochondrial fractions indicated PKA $\alpha$ Cat is located predominantly in the outer membrane whereas AKAP79 is present mainly in the contact site fractions.
\end{abstract}

These data indicate that PKA $\alpha$ Cat is present in the cytoplasm, nucleus and mitochondria of placental cells. AKAP79 is also present in human placental mitochondria but there may be anchoring proteins other than AKAP79 responsible for fixing PKA to the outer membrane. PKA may play roles in mitochondrial protein phosphorylation systems in both cytotrophoblast and syncytiotrophoblast.

Keywords: Placenta, Mitochondria, Trophoblast, Protein kinase, AKAP.

\section{INTRODUCTION}

It has been known for some time that protein phosphorylation by protein kinases is an important component of cellular signalling systems that are located in the plasma membrane, nucleus and cytoplasm [1]. Relatively little is known, however, about cellular signalling from these regions to the mitochondria but several groups have explored the hypothesis that protein phosphorylation plays an important role in the signalling systems that operate in the mitochondrion [26]. In many tissues it is known that protein kinases phosphorylate and activate key proteins such as receptors, enzymes and ion channels and these can in turn then affect cellular functions such as metabolism, differentiation and endocrine functions $[1,2]$. One of the most common protein kinases, cAMP-dependent protein kinase (PKA) can migrate to many areas of the cell and is often held in a precise cellular location by A kinase anchoring proteins (AKAPs) [7]. There are several forms of AKAP (often differentiated by a suffix corresponding to their molecular weight) that have been found in tissues and species other than placenta and human. The only form of AKAP found in human reproductive tissue prior to this investigation was AKAP79 which, was shown to be present in the cytoplasm of human

\footnotetext{
*Address correspondence to this author at the School of Biological Sciences, The University of Sydney, NSW, 2006, Australia;

Tel: +61 29036 6412; Fax: +61 29351 4771;

E-mail: murray.thomson@sydney.edu.au
}

myometrium and is thought to anchor PKA to the myometrial plasma membrane [8].

PKA is activated when cAMP binds to the two regulatory (Reg) subunits, which disengage to release consequently active catalytic (Cat) subunits. There are three known isoforms of the Cat subunit; these are $\alpha$ Cat, $\beta$ Cat, and $\gamma$ Cat and are four known isoforms of the Reg subunit, these are type I $\alpha$ Reg, I $\beta$ Reg, II $\alpha$ Reg and II $\beta$ Reg [9]. PKA is only active when cAMP binds to the Reg subunits, causing Cat subunits to dissociate as free and active units [10] and migrate to different areas of the cell [11]. PKA components have been found in the mitochondria of some species and tissues [2], for example, in the mouse oocyte [12].

PKA enzyme activity has been found in the mitochondria of human placenta and the enzyme appears to contribute to the phosphorylation of a $20 \mathrm{kDa}$ mitochondrial protein (MP20). Modulation of protein phosphorylation in the mitochondria may regulate the physiological processes that mitochondria are involved in, in different tissues such as apoptosis, ATP generation and steroid hormone synthesis $[2,13]$. The finding of PKA enzyme activity in placental mitochondria led to the speculation that PKA is secured in this location by an AKAP [2]. Furthermore, it has been suggested that this placental and mitochondrial PKA may phosphorylate and activate important modulators of placental physiology such as the Steroidogenic Acute Regulatory protein (StAR) $[2,14]$. Such an activation of StAR may be able to increase StAR's regulation of cholesterol transport from the 
outer mitochondrial membrane to the inner membrane where it is converted into progesterone in the first and rate-limiting step in steroidogenesis [15-17].

A vital developmental event for the placenta is the differentiation of trophoblast cells into cytotrophoblast and syncytiotrophoblast [18]. The placental steroid hormones that are vital for pregnancy to progress successfully to term are produced in the syncytiotrophoblast [19]. Mitochondria from the syncytiotrophoblast are lighter than those of the cytotrophoblast and therefore centrifugation can be used to separate the organelles from either cell type of cell $[20,21]$. In this study we used specific antibodies to investigate the possibility that PKA $\alpha$ Cat and AKAP79 are present in various locations in the human placenta including mitochondria from both cytotrophoblast and syncytiotrophoblast.

\section{MATERIALS \& METHODS}

\section{Isolation of Mitochondria from Human Placenta}

Human term placentae were collected from the Royal Prince Alfred Hospital, Sydney, and perfused in chilled phosphate saline buffer (PBS) to remove blood. Mitochondria were isolated as described by Corso and Thomson [21], briefly, the placentae were placed in chilled PBS and the tissues from the maternal side of the placenta were diced into pieces of approximately $1 \mathrm{~cm}^{3}$. The diced tissues were homogenised in homogenisation buffer containing: $20 \mathrm{mM}$ Tris$\mathrm{HCl}, 210 \mathrm{mM}$ mannitol, and $70 \mathrm{mM}$ sucrose. The homogenate was then centrifuged (Beckman J2-21M/E Centrifuge) at 700 $\times \mathrm{g}$ for 10 minutes at $4^{\circ} \mathrm{C}$ to remove cell debris and nuclei, for brevity the pellet from this stage will be referred to as nuclei. The supernatant was decanted and stored at $4^{\circ} \mathrm{C}$, and the pellet was resuspended twice using the homogenisation buffer and centrifuged at $700 \times \mathrm{g}$ for 10 minutes at $4^{\circ} \mathrm{C}$. The combined supernatants were centrifuged at $9750 \times \mathrm{g}$ for 15 minutes at $4^{\circ} \mathrm{C}$ to yield the mitochondrial pellet. The mitochondria were washed free of microsomes and cytosol in the supernatant three times by re-suspension, using a dounce homogenizer, in homogenisation buffer and centrifuged at $9750 \times \mathrm{g}$ for 15 minutes at $4^{\circ} \mathrm{C}$. For brevity the supernatant at this stage will be referred to as microsomes. After three washes the supernatants were removed and the mitochondria pellet was re-suspended in the homogenisation buffer. This preparation is referred to as mitochondria.

\section{Separation of Heavy and Light Mitochondria}

Isolated mitochondria from human placentae were separated into heavy mitochondria characteristic of cytotrophoblast mitochondria and light mitochondria characteristic of syncytiotrophoblast mitochondria by sequential centrifugation following the method of Martinez and colleagues [20]. Briefly, mitochondria were resuspended in an equal volume of homogenisation buffer and centrifuged at $4000 \times$ $\mathrm{g}$ for 15 minutes at $4^{\circ} \mathrm{C}$. The pellet was designated as heavy mitochondria. The pellet was removed and the supernatant was further centrifuged at $16,000 \times \mathrm{g}$ for 15 minutes at $4^{\circ} \mathrm{C}$ to obtain light mitochondria.

\section{Fractionation of Mitochondria}

To produce vesicles of inner membrane, outer membrane and vesicles of outer and inner membrane joined by a contact site, whole unbroken mitochondria were processed by an established swelling and shrinking procedure and sucrose gradient separation technique [22-24]. Briefly, mitochondria in homogenisation buffer were centrifuged at $9750 \times \mathrm{g}$ for 15 minutes at $4^{\circ} \mathrm{C}$ to remove the excess buffer then subjected to swelling and shrinking by resuspension and incubation in $7.5 \mathrm{ml}$ of $10 \mathrm{mM}$ phosphate buffer at $4^{\circ} \mathrm{C}$ for 20 minutes, followed by the addition of $3 \mathrm{ml}$ of $60 \%$ sucrose and further incubated at $4^{\circ} \mathrm{C}$ for 20 minutes. Mitochondrial membranes were further disrupted by sonication using Sonifier B-12 (Branson Sonic Power Company) three times for 30 seconds at level 6 with 30 second intervals in between. Unbroken mitochondria were removed by centrifugation (using Beckman $\mathrm{J} 2-21 \mathrm{M} / \mathrm{E}$ Centrifuge) at $9750 \times \mathrm{g}$ for 20 minutes at $4^{\circ} \mathrm{C}$. This process produces vesicles of inner membrane that are generally relatively large, smaller vesicles of outer membrane and vesicles of outer membrane joined at a contact site to a vesicle of inner membrane and these complexes are mainly intermediate in size. $5 \mathrm{ml}$ aliquots of suspensions of vesicles (with protein concentration of approximately $5 \mathrm{mg} / \mathrm{ml}$ ) were layered on a $23 \mathrm{ml}, 24.4 \%-61.2 \%$ continuous sucrose density gradient. Sucrose gradients were subjected to ultracentrifugation (using Beckman XL-90 Ultracentrifuge) at $100,000 \times \mathrm{g}$ for 20 hours at $4^{\circ} \mathrm{C}$. The gradient was then collected as 20 fractions of $1.4 \mathrm{ml}$ each, starting at the bottom of the tube. The majority of inner membrane is found at the bottom of the tube, the outer membrane is located mainly towards the top of the tube and the contact sites are located between the inner and outer membrane [22, 23, 16, 24]. The positions of outer and inner membrane were checked to be the same as previous implementations of this procedure using enzyme markers [24].

\section{Protein Concentration Estimation}

The protein concentration of samples from each placenta was assayed using the Pierce Bicinchoninic acid (BCA) Protein Assay Kit and Bio-Rad Microplate Manager (Version 5.0.1) as per manufacturer instructions.

\section{Sodium Dodecyl Sulphate Polyacrylamide Gel Electro- phoresis (SDS PAGE)}

All samples were diluted so as to contain an equal amount of protein. Samples containing a total of $20 \mu \mathrm{g}$ of protein were loaded onto a $12 \%$ SDS gel and run at a voltage of $100 \mathrm{~V}$ using the Biorad Protean III System according to the manufacturer until the bromophenol blue dye font reached the bottom of the gel (approximately 2 hours). For sucrose gradient samples the first 19 fractions were loaded leaving one lane free for standard molecular weight markers. Protein bands were detected using Coomassie blue stain. Where a representative single lane from several repeats is shown in a diagram, the lane shown is that of the first sample analysed. There was no post result selection of lanes for diagrams.

\section{Western Blotting}

Proteins were transferred to nitrocellulose membrane (100V for 1 hour) using a Mini Trans-Blot system (Bio-Rad laboratories) according to manufacturer's instructions. Complete transference of colour coded molecular weight stan- 
dards from the gel to the nitrocellulose membrane was checked. The nitrocellulose membrane was blocked with 5\% skim milk in $1 \%$ phosphate-buffered saline (1\% PBS) for 1 hour. Following the blocking of non-specific binding the nitrocellulose membrane was incubated with primary antibody for 1 hour. The primary antibodies used were PKA $\alpha$ Cat (C-20) rabbit polyclonal IgG antibody (Santa Cruz) and AKAP79 (H-105) rabbit polyclonal IgG antibody (Santa Cruz). The membranes were given 2 quick rinses in phosphate-buffered saline containing $0.05 \%(\mathrm{v} / \mathrm{v})$ Tween-20 (TPBS), $4 \times 5$ minutes washes with TPBS, and 30 minutes incubation in secondary antibody. The membrane was then given 2 quick rinses in TPBS, $4 \times 5$ minutes washes in TPBS, 1 rinse in PBS and then incubated in chemiluminescent substrate (Pierce SuperSignal West Pico) as per the manufacturer's instructions. The membranes were then exposed to film (Pierce Cl-Xposure) for various times and were analysed using the public domain computer program ImageJ, a Java conversion of NIH Image developed at the U.S. National Institutes of Health and available on the Internet at http://rsb.info.nih.gov/nih-image/).

\section{Statistical Analysis}

Results were analysed by paired t-test assuming equal variance and a $P$ value of 0.05 or less was considered significant.

\section{RESULTS}

\section{Isolation of Cellular Fractions from Human Placenta}

Proteins in the, crude homogenate, nuclei, microsomes and mitochondria from three different human placentae were analysed using SDS PAGE and detected using Coomassie blue (Fig. 1). The homogenate, nuclei, microsomes and mitochondria showed protein band patterns, which spanned the
7-215 kDa molecular weight range. Densitometry analysis of the bands displayed in Fig. (1A) showed that the total placenta homogenate and microsomes displayed major peaks at 65 and $10 \mathrm{kDa}$ that were not present in mitochondrial fractions (Fig. 1B).

\section{PKAa Cat expression in Human Placental Tissue}

When Western blots of nuclei, microsomes and mitochondria were probed with the PKA $\alpha$ Cat antibody they all displayed a major band of immunoreactivity at approximately $44 \mathrm{kDa}$ (Fig. 2). A minor band was observed at an approximately $90 \mathrm{kDa}$. One sample of each cellular fraction from three different placentae was analysed, and similar results were observed in three different human placentae (Fig. 2). The intensity of the $44 \mathrm{kDa}$ PKA $\alpha$ Cat band in microsome fractions was significantly higher than in mitochondria $(p=0.02)$. On the other hand, there was no significant difference in the band intensity between nuclei and mitochondria $(p=0.30)$, and nuclei and microsomes $(p=0.23)$.

\section{PKA $\alpha$ Cat expression in Heavy And light Mitochondria}

To determine the difference in the level of PKA $\alpha$ Cat expression in heavy and light placental mitochondria, isolated mitochondria were separated into heavy and light mitochondria as described in methods and materials. Each placenta (of a total of 5) yielded one isolate of heavy mitochondria and one isolate of light mitochondria. From each isolate (from each placenta) four aliquots were taken for SDS PAGE analysis and Western blotting. The process was repeated a total of five times, each of these times using a different placenta.

The intensities of the PKA $\alpha$ Cat immuno-reactive bands at $44 \mathrm{kDa}$ in heavy and light mitochondria were compared for isolates from each placenta, (Fig. 3). There was no sig-

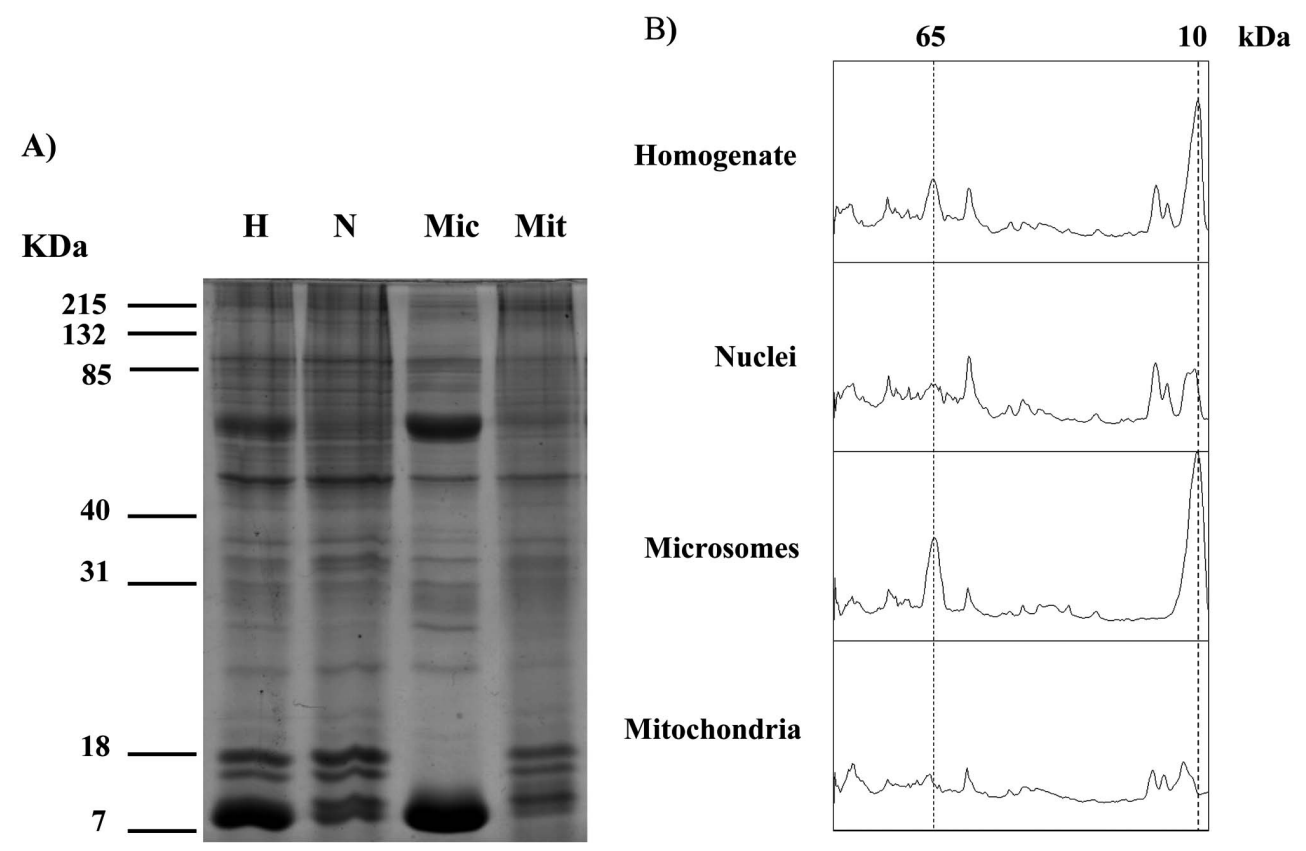

Fig. (1). SDS PAGE analysis of proteins in homogenate (H), nuclei (N), microsomes (Mic) and mitochondria (Mit) fractions from human placenta. Similar results were observed in a total of 3 different placentae. Gels stained with Coomassie are shown in A), densitometry scans of the lanes are shown in $\mathbf{B}$ ) the positions at $65 \mathrm{kDa}$ and $10 \mathrm{kDa}$ are indicated with dotted lines. 

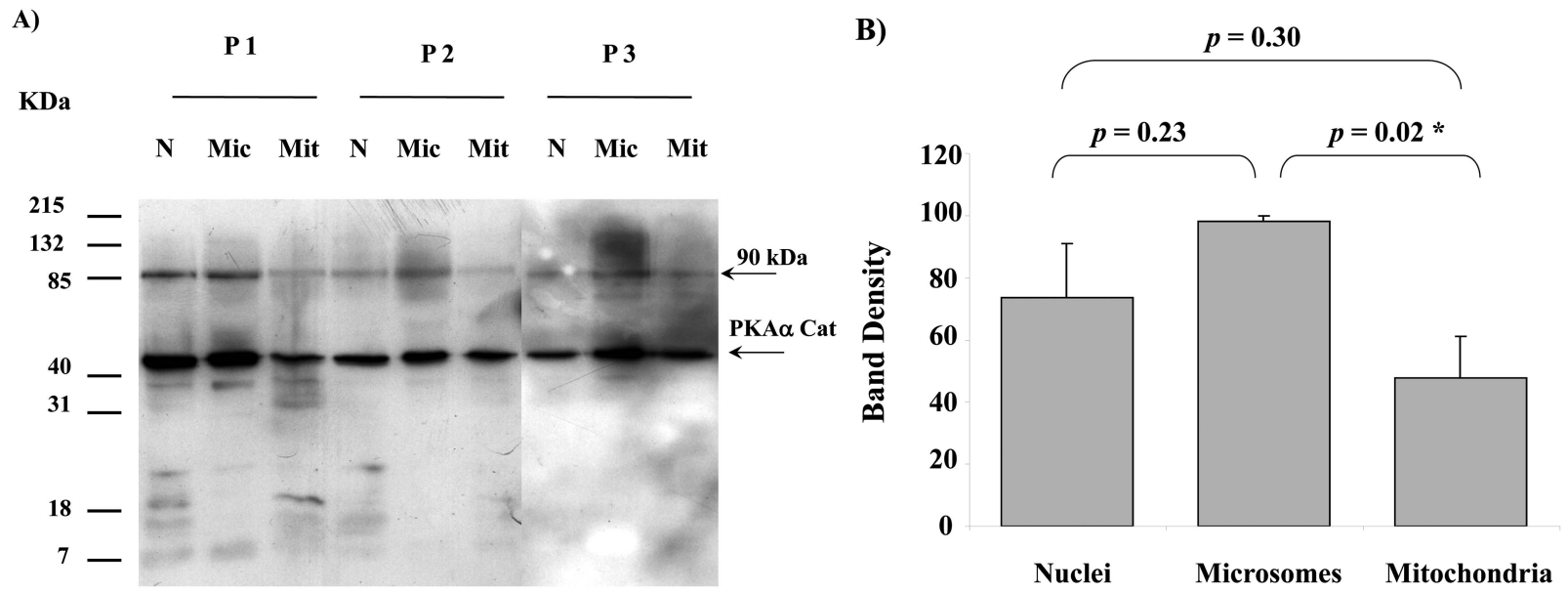

Fig. (2). PKA $\alpha$ Cat expression in nuclei (N), microsomes (Mic) and mitochondria (Mit) from 3 different human placentae, (P1, P2 and P3). For each placenta one sample from each fraction was analysed. SDS PAGE, Western blotting and autoradiography results shown in A). A major protein band with approximate size of $44 \mathrm{kDa}$ was detected in all fractions by the PKA $\alpha$ Cat antibody. A minor band at approximately $90 \mathrm{kDa}$ was also present. The relative intensity taken as percentage of the most intense band for each placenta (values represent the mean \pm S.E.) of the $44 \mathrm{kDa}$ bands shown in A) are plotted in B). An asterisk indicates a significant difference with a $\mathrm{p}$ value of less than 0.05 .
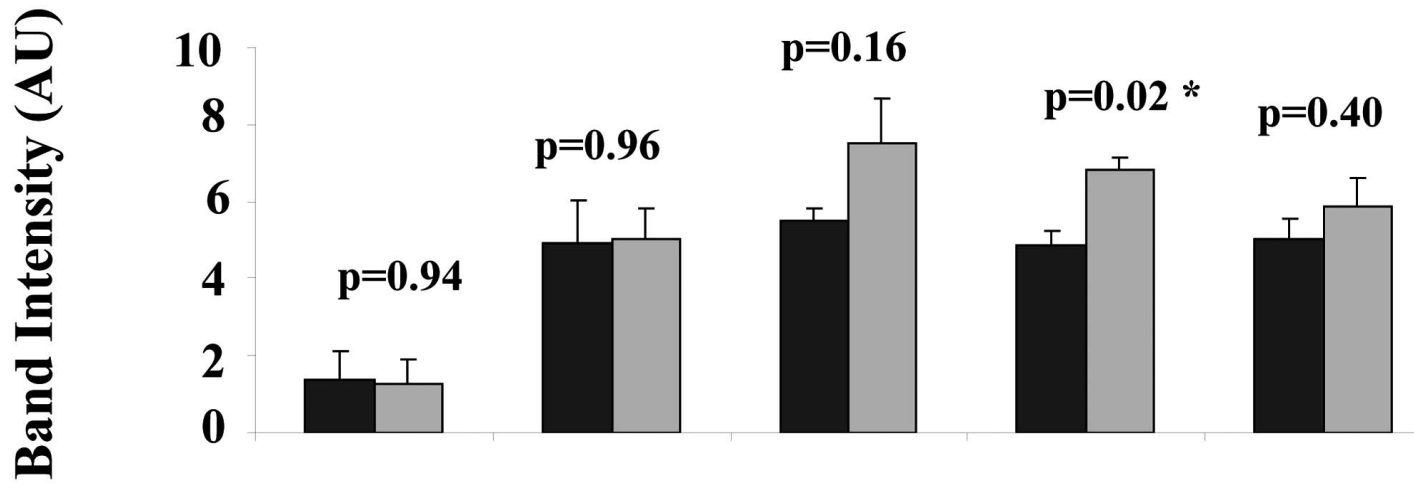

Fig. (3). Comparison of PKA $\alpha$ Cat expression in heavy (H) and light (L) mitochondria. For each of 5 placentae one preparation of heavy and one preparation of light mitochondria was produced. For each preparation of light or heavy mitochondria, three aliquots were taken for SDS PAGE and Western blot, the intensities of the three $44 \mathrm{kDa}$ protein bands in each preparation of heavy and light mitochondria from five different human placentae are shown. Values represent the mean \pm S.E. of triplicate samples $\square-$ heavy mitochondria; $\square-$ light mitochondria. An asterisk indicates a significant difference with a $\mathrm{p}$ value of less than 0.05 .

nificant difference in the band intensity for the $44 \mathrm{kDa} P K A \alpha$ Cat immuno-reactive band between the heavy and light mitochondria in four out of five human placentae $(p=0.94$ for placenta $1, \mathrm{p}=0.96$ for placenta $2, \mathrm{p}=0.16$ for placenta $3, \mathrm{p}$ $=0.40$ for placenta 5 ). There was a significant difference in the band intensity for the $44 \mathrm{kDa}$ PKA $\alpha$ Cat immunoreactive band between the heavy and light mitochondria in human placenta $4(\mathrm{p}=0.02)$.

\section{AKAP79 Expression in Human Placental Tissue}

Western blots of nuclei, microsomes and mitochondria all displayed a major band of AKAP79 immunoreactivity at the approximately $80 \mathrm{kDa}$ region using AKAP79 antibody (Fig. 4). The intensity of this protein band detected in nuclei, microsomes and mitochondria from three different human placentae are shown in Fig. (4B). There was no significant difference in the band intensity between nuclei, microsomes and mitochondria (nuclei and microsomes: $\mathrm{p}=0.18$; nuclei and mitochondria: $\mathrm{p}=0.89$; microsomes and mitochondria: $\mathrm{p}$ $=0.09)($ Fig. 4B).

\section{AKAP79 Expression in Heavy and Light Placental Mito- chondria}

To determine the difference in the level of AKAP79 expression in heavy and light placental mitochondria, isolated mitochondria were separated into heavy and light mitochondria as described in methods and materials. Each placenta (of a total of 5) yielded one isolate of heavy mitochondria and one isolate of light mitochondria. From each isolate (from each placenta) four aliquots were taken for SDS PAGE analysis and Western blotting. The process was repeated a total of five times, each of these times using a different placenta. The AKAP79 immuno-reactive band was present in both heavy and light mitochondria in all placentae (Fig. 5). The band intensity of the AKAP79 band was not significantly different as compared between the heavy and light placental mitochondria in any of the human placenta $(p=$ 

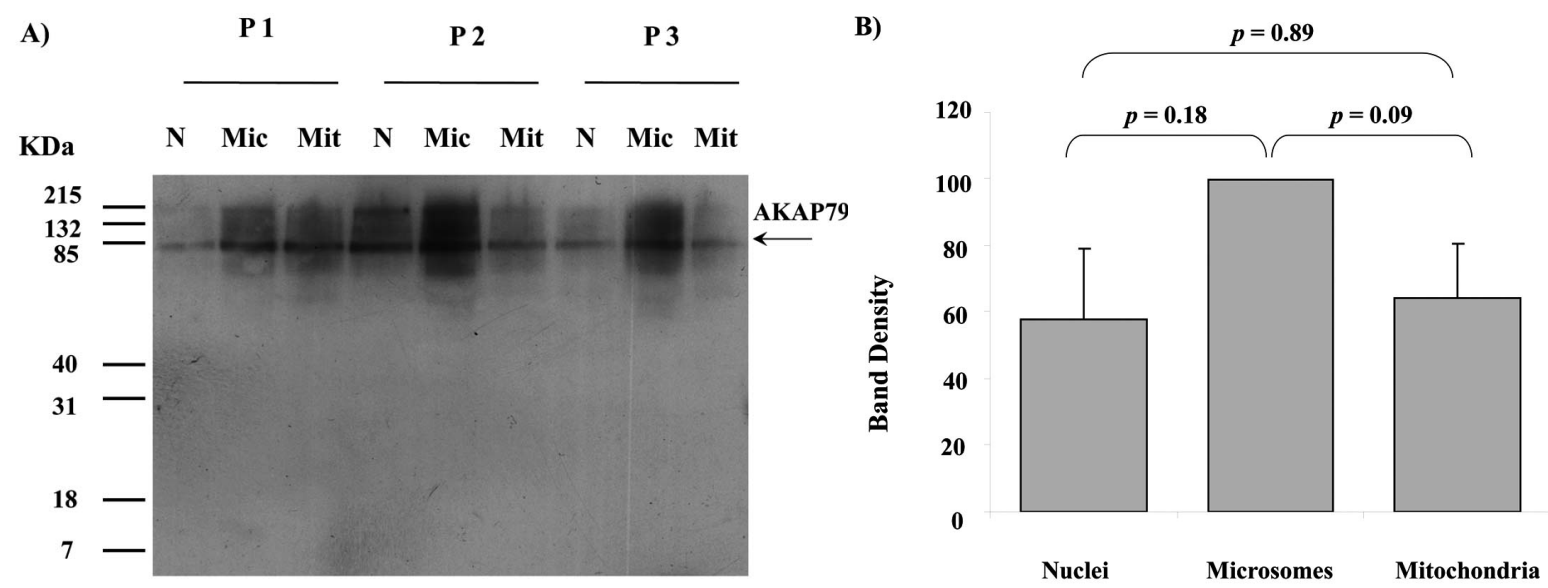

Fig. (4). AKAP79 immunoreactivity in nuclei (N), microsomes (Mic) and mitochondria (Mit) from 3 different human placentae, (HP 1, 2 and 3). SDS PAGE, Western blotting and autoradiography results shown in a). A protein band of approximate size $80 \mathrm{kDa}$ was detected in all fractions by the AKAP79 antibody (A). The relative intensity taken as percentage of the most intense band for each placenta (values represent the mean \pm S.E.) of the $80 \mathrm{kDa}$ bands shown in $\mathbf{A}$ ) are plotted in $\mathbf{B}$ )

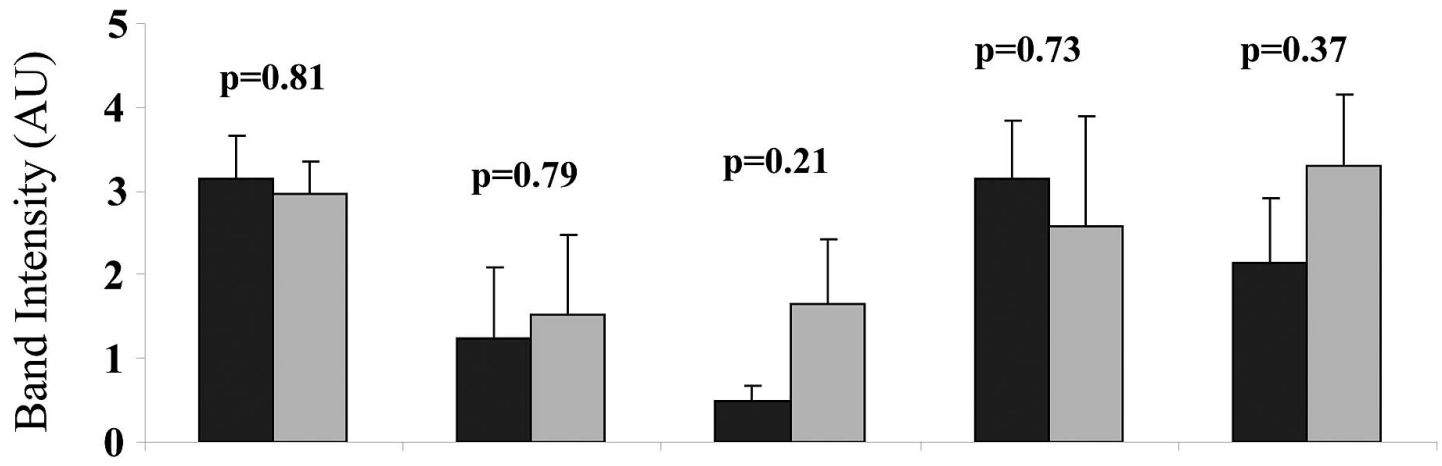

Fig. (5). Comparison of the AKAP79 expression in the heavy (H) and light (L) mitochondria. For each of 5 placentae one preparation of heavy and one preparation of light mitochondria was produced. For each preparation of light or heavy mitochondria, three aliquots were taken for SDS PAGE and Western blot. The intensities of the AKAP79 immuno-reactive protein bands in each preparation of heavy and light mitochondria from five different human placentae are shown. Values represent the mean \pm S.E. of triplicate samples $\mathbf{a}-$ heavy mitochondria; - light mitochondria.

0.81 for placenta $1, \mathrm{p}=0.79$ for placenta $2, \mathrm{p}=0.21$ for placenta $3, \mathrm{p}=0.73$ for placenta $4, \mathrm{p}=0.37$ for placenta 5 ).

\section{PKAa Cat Immunoreactivity in Human Placental Submi- tochondrial Fractions}

When submitochondrial fractions were separated on sucrose gradients and analysed using SDS-PAGE and Western blot, PKA $\alpha$ Cat immuno-rearctivity peaked at fraction 17 in the outer membrane region of the gradient (Fig. 6).

\section{AKAP79 Cat Expression in Human Placental Submitochondrial Fractions}

When submitochondrial fractions were separated on sucrose gradients and analysed using SDS-PAGE and Western blot, AKAP79 immuno-reactivity peaked at fraction 12 in the contact site region of the gradient (Fig. 7).

\section{DISCUSSION}

The method used in this study to fractionate cellular components has been shown to produce mitochondria free of any significant contamination from microsomes using the ras protein as an indicator, ras is present in the microsome fraction but is undetectable in the mitochondria fraction [25]. In the present study we again checked the purity of the mitochondrial fraction using the 65 and $10 \mathrm{kDa}$ proteins that are present in the microsome and homogenate fractions but absent from the mitochondria fraction (Fig. 1).

Prior to this study PKA enzyme activity has been shown to be present in human placental mitochondria [21] but whether this bioactivity was exerted by a version of PKA that contained an $\alpha$ catalytic subunit was not known. Indeed there has been no prior study that has shown what PKA subunits are present in any cellular location in human placenta. In this study the presence of PKA $\alpha$ Cat positive bands of immunoreactivity in all three cellular subfractions studied (Fig. 2A) indicates that PKA $\alpha$ Cat is ubiquitous in the human placental cells and that levels of PKA $\alpha$ Cat in the mitochondria are of similar concentrations to that found in the nuclei (Fig. 2B).

The molecular weight estimation by SDS PAGE of approximately $44 \mathrm{kDa}$ for PKA $\alpha$ Cat is the same reported by the antibody manufacturer when detecting PKA $\alpha$ Cat in mouse brain extract (http://datasheets.scbt.com/sc-903.pdf) 


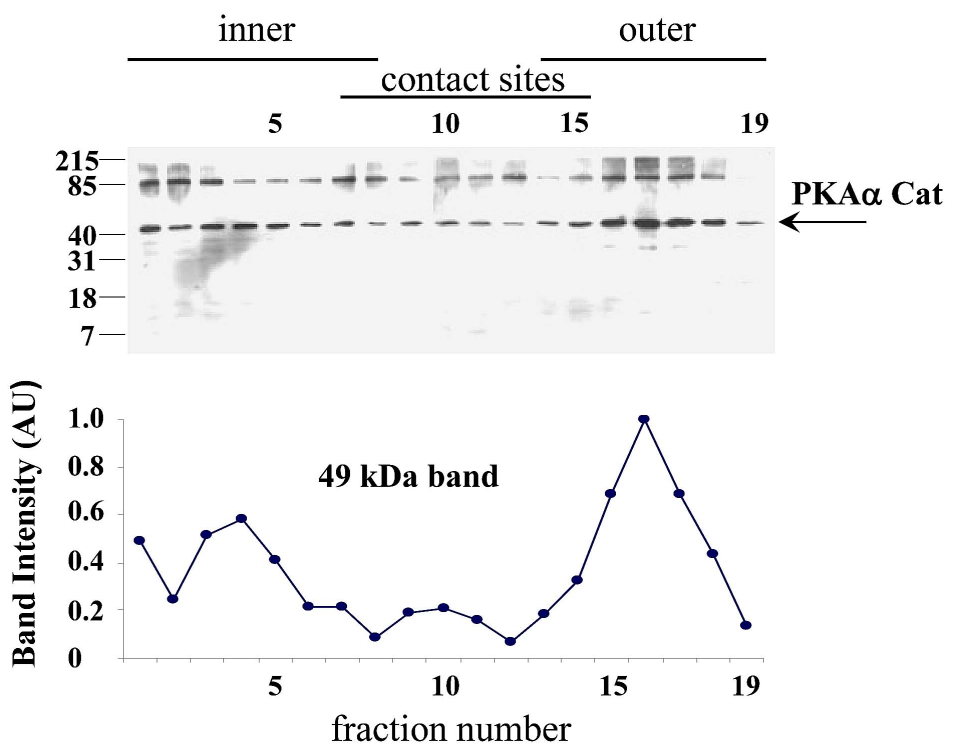

Fig. (6). PKA $\alpha$ Cat expression in the human placental mitochondrial fractions. PKA $\alpha$ Cat expression was detected using SDS-PAGE, Western blotting and autoradiography (Exposure time $=1$ minute). The PKA $\alpha$ Cat immuno-reactive bands are shown in $\mathbf{A}$ ), the intensities of the $44 \mathrm{kDa}$ PKA $\alpha$ Cat bands are shown in B). The experi-ment was repeated for a total of three different placentae with similar results.

A)

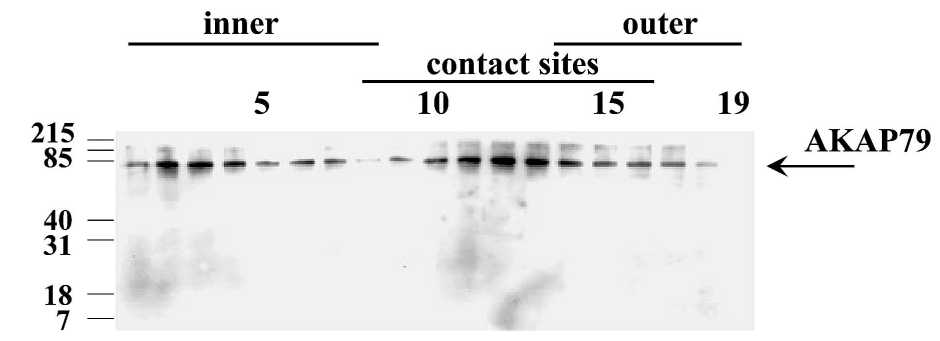

B)

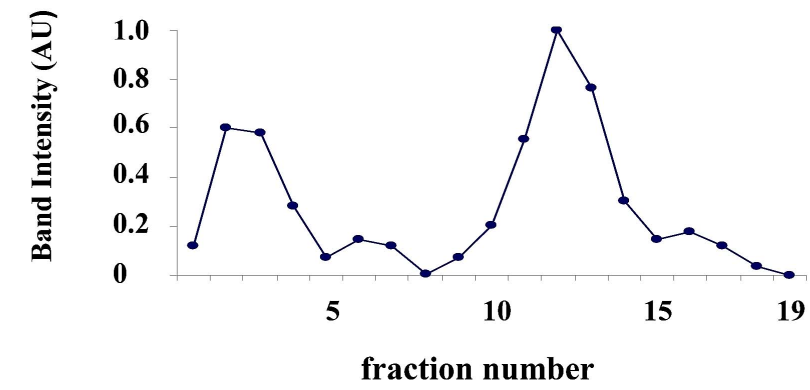

Fig. (7). AKAP79 immunoreactivity in the human placental mitochondria fractions. AKAP79 immunoreactivity was detected using SDSPAGE, Western blotting and autoradiography (Exposure time $=1$ minute). The AKAP79 immuno-reactive bands are shown in A) the intensity of the AKAP79 bands are shown in B). The experiment was repeated for a total of three different placentae with similar results.

and similar to the estimated molecular weight of PKA $\alpha$ Cat previously found in the cytoplasm of human myometrium (approximately $42 \mathrm{kDa}$ ) [8]. SDS PAGE has an accuracy in molecular weight determination of up to approximately +/$10 \%$ [26]. Interestingly, an additional minor PKA $\alpha$ Cat band at $90 \mathrm{kDa}$ was detected by the PKA $\alpha$ Cat antibody in mitochondria as well as nuclei and microsomes (Fig. 2). The minor $90 \mathrm{kDa}$ band may indicate some aggregation of PKA $\alpha$ Cat or the presence of a larger weight precursor protein.

Cytotrophoblast mitochondria are heavier than syncytiotrophoblast mitochondria [20] and the finding that the level of PKA $\alpha$ Cat immunoreactivity was not significantly different in light mitochondria as compared to heavy mitochondria in four out of five placentae tested (Fig. 3) suggests that syncytiotrophoblast mitochondria do not need significantly more PKA than cytotrophoblast mitochondria for physiological uses of PKA in the organelle. Previous studies using phosphotyrosine antibodies have demonstrated that while some phosphotyrosine proteins are evenly distributed between heavy and light mitochondria, the concentrations of two phosphoproteins of 20 and $16 \mathrm{kDa}$ molecular weight was significantly higher in heavy mitochondria. Additionally, the amount of porin, the protein that makes up the voltage dependent anion channel in mitochondria is significantly higher in heavy mitochondria (Ma and Thomson unpublished results). It will be interesting to determine in future studies 
what physiological roles PKA is playing in the mitochondria of both syncytiotrophoblast and cytotrophoblast.

For PKA to be involved in physiological processes in the mitochondria of human placenta it would be expected that an AKAP would be present in the organelle to fasten the protein in the organelle [2]. Figs $(\mathbf{4}, \mathbf{5})$ and $(\mathbf{7})$ indicate that human placental mitochondria contain at least one AKAP, AKAP79 at concentrations that are similar to those found in the nuclei and microsome fractions (Fig. 4). This AKAP79 displays a similar migration distance in SDS PAGE compared to AKAP79 in other cells such as neuroblastoma and myoblast cell lines [27]. Both cytotrophoblast and syncytiotrophoblast mitochondria appear to contain this AKAP at similar concentrations and future studies may reveal the role played by AKAP79 in both types of mitochondria. Fig. (5) indicates that both heavy and light mitochondria contain similar amounts of AKAP79 immunoreactivity and it will be important in future investigations to determine whether AKAP79 is binding PKA subunits with active enzyme activity in both heavy and light mitochondria of human placenta.

The pattern of PKA $\alpha$ Cat immunoreactivity in sucrose gradient separations of submitochondrial fractions suggests that PKA $\alpha$ Cat is located predominantly in the outer membrane (Fig. 6). AKAP79 on the other hand appears to be located predominantly in contact site vesicles (Fig. 7) and so it appears that the majority of AKAP79 is not responsible for anchoring the majority of PKA $\alpha$ Cat in these mitochondria. It is possible that another species of AKAP may be anchoring substantial quantities of the PKA in placental mitochondria. Submitochondrial fractionation on sucrose gradients are useful to show where the majority of a protein is in the mitochondrion but because of the heterogeneity in size of submitochondrial particles the technique cannot fully purify inner membrane from outer membrane and cannot quantify the relative amounts of a protein in different mitochondrial locations (see Thomson for review) [16]. Therefore the possibility remains that smaller amounts of AKAP79 in the outer membrane may contribute to the anchoring of PKA in this region.

Indeed, there is a paucity of information on the protein kinase and AKAP species that are present in the human placenta and it will be important in future studies to determine what combinations and configurations of kinase subunits and anchors are present in the cells and sub-cellular locations of the organ. Future experiments using electron microscopy and immuno-gold labelling [28] may help to show what AKAP species are co-localised with PKA in the mitochondria. In some steroidogenic tissues phosphorylation of the steroidogenic acute regulatory protein (StAR) in or close to the mitochondria by PKA is thought to accelerate cholesterol transport to the inner membrane for conversion to pregnenolone [29-32] and AKAP121 appears to facilitate steroidogenesis in the mouse leydig cell line MA-10 [33].

\section{CONCLUSION}

This study has indicated that PKA $\alpha$ Cat and AKAP79 are present in the mitochondria of both cytotrophoblast and syncytiotrophoblast. PKA $\alpha$ Cat it is located mainly in the outer membrane whereas AKAP79 is located predominantly in the contact sites. Future experiments are necessary to es- tablish the physiological roles of PKA $\alpha$ Cat and AKAP79 in the mitochondria of human placenta.

\section{CONFLICT OF INTEREST}

None declared.

\section{ACKNOWLEDGEMENTS}

We would like to thank the staff of the Women and Babies Service at The Royal Prince Alfred Hospital for help in collecting the placentae. This study was conducted under the Royal Prince Alfred Hospital Human Ethics Committee protocol X06-0061 and funded by a grant from the Integrative Physiology Group, The University of Sydney.

\section{ABBREVIATIONS \\ AKAP79 $=$ A Kinase Anchoring Protein of approxi- mate molecular weight $79 \mathrm{kDa}$ \\ $\mathrm{BCA}=$ Bicinchoninic acid \\ Cat $=$ Catalytic \\ MP20 $=$ Mitochondrial protein of $20 \mathrm{kDa}$ \\ OCT $=$ Optimal cutting temperature \\ PBS $=$ Phosphate buffered saline \\ PKA $\alpha$ Cat $=$ Protein Kinase A Subunit $\alpha$ Catalytic \\ Reg $=$ Regulatory \\ StAR $=$ Steroidogenic acute regulatory protein}

\section{REFERENCES}

[1] Pawson, T.; Scott, J.D. Protein phosphorylation in signaling - 50 years and counting. Trends Biochem. Sci., 2005, 30, 286-290.

[2] Thomson, M. Evidence of undiscovered cell regulatory mechanisms: Phosphoproteins and protein kinases in mitochondria. Cell Mol. Life Sci., 2002, 59, 213-219.

[3] Marin-Garcia, J.; Goldenthal, M.J. Heart mitochondria signaling pathways: appraisal of an emerging field. J. Mol. Med., 2004, 82, 565-578.

[4] Horbinski, C.; Chu, C.T. Kinase signaling cascades in the mitochondrion: a matter of life or death. Free Radic. Biol. Med., 2005, 38, 2-11.

[5] Salvi, M.; Brunati, A.M.; Toninello, A. Tyrosine phosphorylation in mitochondria: A new frontier in mitochondrial signaling. Free Radic. Biol. Med., 2005, 38, 1267-1277.

[6] Pagliarini, D.J.; Dixon, J.E. Mitochondrial modulation: reversible phosphorylation takes center stage? Trends Biochem. Sci., 2006, 31, 26-34.

[7] Pawson, T.; Scott, J.D. Signaling through scaffold, anchoring, and adaptor proteins. Science, 1997, 278, 2075-2080.

[8] Ayres, A.W.; Carr, D.W.; McConnell, D.S.; Lieberman, R.W.; Smith, G.D. Expression and intracellular localization of protein phosphatases $2 \mathrm{~A}$ and $2 \mathrm{~B}$, protein kinase $\mathrm{A}$, A-kinase anchoring protein (AKAP79), and binding of the regulatory (RII) subunit of protein kinase A to AKAP79 in human myometrium. J. Soc. Gyn. Invest., 2003, 10, 428-437.

[9] Beebe, S.J. The cAMP-dependent protein kinases and cAMP signal transduction. Sem. Cancer Biol., 1994, 5, 285-294.

[10] Brostrom, M.A.; Reimann, E.M.; Walsh, D.A.; Krebs, E.G. A cyclic 3',5'-AMP-stimulated protein kinase from cardiac muscle. Adv. Enzyme. Regul., 1970, 8, 191-203.

[11] Feliciello, A.; Gottesman, M.E.; Avvedimento, E.V. The biological functions of A-kinase anchor proteins. J. Mol. Biol., 2001, 308, 99114. 
[12] Newhall, K.J.; Criniti, A.R.; Cheah, C.S.; Smith, K.C.; Kafer, K.E.; Burkart, A.D.; McKnight, G.S. Dynamic anchoring of PKA is essential during oocyte maturation. Curr. Biol., 2006, 16, 321-327.

[13] Marin-Garcia, J. Heart mitochondria: A receiver and integrator of signals. New York, Springer, 2011.

[14] Thomson, M. Signal transduction of steroidogenic hormones to the adrenal and gonadal mitochondria and the possibilities for combating lipoid congenital adrenal hyperplasia. Curr. Signal Trans. Ther., 2010, 5, 161-169.

[15] Clark, B.J.; Wells, J.; King, S.R.; Stocco, D.M. The purification, cloning, and expression of a novel luteinizing hormone-induced mitochondrial protein in MA-10 mouse leydig tumor cells Characterization of the steroidogenic acute regulatory protein (StAR). J. Biol. Chem., 1994, 269, 28314-28322.

[16] Thomson, M. Molecular and cellular mechanisms used in the acute phase of stimulated steroidogenesis. Horm. Metab. Res., 1998, 30, $16-28$

[17] Rone, M.B.; Fan, J.; Papadopoulos, V. Cholesterol transport in steroid biosynthesis: Role of protein-protein interactions and implications in disease states. BBA-Mol. Cell Biol. L., 2009, 1791, 646-658.

[18] Huppertz, B. The anatomy of the normal placenta. J. Clin. Pathol., 2008, 61, 1296-1302.

[19] Albrecht, E.D.; Pepe, G.J. Placental steroid hormone biosynthesis in primate pregnancy. Endocr. Rev., 1990, 11, 124-150.

[20] Martinez, F.; Kiriakidou, M.; Strauss, J.F. Structural and functional changes in mitochondria associated with trophoblast dIfferentiation: Methods to isolate enriched preparations of syncytiotrophoblast mitochondria. Endrocrinology, 1997, 138, 2172-2183.

[21] Corso, M.; Thomson, M. Protein phosphorylation in mitochondria from human placenta. Placenta, 2001, 2, 432-439.

[22] Adams, V.; Bosch, W.; Schlegel, J.; Wallimann, T.; Brdiczka, D. Further characterization of contact sites from mitochondria of different tissues: topology of peripheral kinases. BBABiomembranes, 1989, 981, 213-225.
[23] Thomson, M.; Korn, M.; Hall, P.F. GTP-binding proteins in adrenocortical mitochondria. BBA-Protein Struct. M., 1995, 1248, 159-169.

[24] Kuyznierewicz, I.; Thomson, M. GTP-binding proteins $\mathrm{G}_{(\mathrm{s} \alpha)}, \mathrm{G}_{(\mathrm{i \alpha} \alpha}$, and Ran identified in mitochondria of human placenta. Cell. Biol. Int., 2002, 26, 99-108

[25] Thomson, M.; Lim, G.; Hall, P.F.; Kuyznierewicz, I. Overlay blot identification of GTP-binding proteins in mitochondria from human placenta. Placenta, 1998, 19, 209-215.

[26] Weber, K.; Osborn, M. Reliability of molecular weight determinations by dodecyl sulfate-polyacrylamide gel electrophoresis. J. Biol. Chem., 1969, 244, 4406-4412.

[27] Schillace, R.V.; Miller, C.L.; Carr, D.W. AKAPs in lipid rafts are required for optimal antigen presentation by dendritic cells. Immunol. Cell Biol., 2011, 89, 650-658.

[28] Sík, A.; Gulácsi, A.; Lai, Y.; Doyle, W.K.; Pacia, S.; Mody, I.; Freund, T.F. Localization of the A kinase anchoring protein AKAP79 in the human hippocampus. Euros. J. Neurosci., 2000, 12, 1155-1164.

[29] Arakane, F.; King, S.R.; Du, Y.; Kallen, C.B.; Walsh, L.P.; Watari, H.; Stocco, D.M.; Strauss, J.F. Phosphorylation of steroidogenic acute regulatory protein (StAR) modulates its steroidogenic activity. J. Biol. Chem., 1997, 272, 32656-32662.

[30] Petrescu, A.D.; Gallegos, A.M.; Okamura, Y.; Strauss, J.F.; Schroeder, F. Steroidogenic acute regulatory protein binds cholesterol and modulates mitochondrial membrane sterol domain dynamics. J. Biol. Chem., 2001, 276, 3697-36982.

[31] Stocco, D.M. StAR protein and the regulation of steroid hormone biosynthesis. Annu. Rev. Physiol., 2001, 63, 193-213.

[32] Thomson, M. Does cholesterol use the mitochondrial contact site as a conduit to the steroidogenic pathway? Bioessays, 2003, 25, 252258.

[33] Dyson, M.T.; Jones, J.K.; Kowalewski, M.P.; Manna, P.R.; Alonso, M.; Gottesman, M.E.; Stocco, D.M. Mitochondrial A-kinase anchoring protein 121 binds type II protein kinase A and enhances steroidogenic acute regulatory protein-mediated steroidogenesis in MA-10 mouse Leydig tumor cells. Biol. Reprod., 2008, 78, 267277.

(C) Ma and Thomson; Licensee Bentham Open.

This is an open access article licensed under the terms of the Creative Commons Attribution Non-Commercial License (http://creativecommons.org/licenses/by-nc/3.0/) which permits unrestricted, non-commercial use, distribution and reproduction in any medium, provided the work is properly cited. 